\title{
ADVANTAGES OF BREATHING EXERCISES ON PIANO PERFORMANCE FOR BEGINNERS
}

Salwa Hassan Mohamed Mahmoud PASHA *

Music Education Department, Faculty of Specific Education, Alexandria University, Egypt

\begin{abstract}
Breathing is a vital process, as mentioned by the famous pianist Andras Schiff. Often pianists forget to breathe while performing on the piano keyboard in the style of music output, which negatively affects them and they become tense and have physical pain. The performance on the piano keyboard for beginners requires mental conditioning instead of conditioning the muscles to gain the skilful ability to withstand the performance of various techniques in a graded manner without fatigue or excessive tension, as the body needs an adequate amount of oxygen and adequate blood flow to the muscles and some organs and the brain, Meditation, with a focus on proper breathing using the diaphragm and body positions, is the largest factor that affects the amount of oxygen with lung efficiency.

Keywords

Advantages, Breathing Exercises, Piano Performance, Beginners.
\end{abstract}

\section{Introduction}

The researcher noticed through teaching that novice students do not remember taking the breath correctly, which causes an increase in psychological and physical tension during performance on the piano keyboard, which leads to the occurrence of technical musical errors and it is difficult for the novice to control the balanced touch of the fingers on the fingers of the plate The keys of the piano machine, so the researcher believed that teaching self exercises in a correct manner can help beginners to perform well and control the balanced touch of the fingers of the piano keyboard.

The research is divided into two parts:

The first part: the theoretical framework and includes:

Firstly, previous studies related to the research topic:

The first study: The relationship between musical characteristics and temporal breathing pattern in piano performance.

Relationship between Musical Characteristics and Temporal Breathing Pattern in Piano Performance

The study stated that despite increasing evidence that breathing is shaped by motor and cognitive activities, the nature of breathing in musical performance has been little explored. So, in the current study, the researcher presented a temporal breathing pattern in piano performance, with the aim of demonstrating how breathing timing relates to musical organization / events and performance. The results indicate the different performances of

* Corresponding author: spedu@alexu.edu.eg 
pianists from (amateur professionals) to ten musical excerpts, with different individual differences, scientific and practical experiences for each of them, and the nature of the musical scores presented through an analysis of the relationship between respiration and musical characteristics.

Study 2: Dealing with Performance Anxiety: Piano students' perceptions of speaking about performance anxiety and the potential effectiveness of deep breathing, deep muscle relaxation, and visualization.

Coping with Performance Anxiety: College Piano Students' Perceptions of Performance Anxiety and Potential Effectiveness of Deep Breathing, Deep Muscle Relaxation, and Visualization.

The study aimed to sensitize piano students to the pre-management of performance anxiety with the use of deep breathing, deep muscle relaxation, and visualization as a self-regulating technique that may contribute to the alleviation of psychological and physiological symptoms associated with performance anxiety. This technique was presented in a workshop on how to control the anxiety faced by performance participants through a series of deep breathing exercises, deep muscle relaxation and visualization. The surveys and results also revealed that experienced pianists, whether they played alone or in groups, have the ability to control the level of anxiety about less experienced pianists, as the largest percentage of workshop participants are musicians who indicated that they use deep breathing and Positive thinking as a self-organizing technique is considered a type of effective strategy in dealing with anxiety during performance.

Second: The theoretical framework:

Respiratory system.

It is considered one of the body systems most important in the science of respiration as a source of human life. The respiratory system is formed according to the order of entry of the inhaled air as follows (nose - nasopharynx - oropharynx - larynx - trachea, bronchi - bronchioles alveoli inside the lungs, which are located inside the rib cage). In addition, an important muscle that has an effective role in breathing (diaphragm muscle) is added. The nose: an organ in the middle of the human face that has two openings between them, there is a bone barrier, and inside the two openings there are thin hairs that help in the process of breathing in the air of impurities, and inside the nose there are sinuous cartilages lined with a sensitive mucous membrane filled with nerves and capillaries that warm the inhaled air coming from outside The mouth becomes equal to body temperature. Nasopharynx and oropharynx: It consists of a soft membrane that lines the inner wall of the nose and mouth and then connects to the esophagus. 
Larynx: It consists of three cartilages, linked by different ligaments, and within it are the vocal folds responsible for producing the sound. Trachea: a tube composed of cartilages in the form of semicircular rings that begins with the cricoid cartilage, which is the base of the larynx. Its rings are connected by membranous tissue whose surface cells are ciliary, and the diameter of the trachea is between two to two and a half centimeters and contains from sixteen to eighteen cartilages It is between ten to twelve centimeters in length and is divided from the bottom into two main branches:

The bronchi are two cylindrical channels branching from the trachea, each of which branches into several small bronchi, each of which ends in one of the alveoli in which gases are exchanged and blood oxidation takes place.

The lungs: The lungs are located inside the rib cage in the human chest, as each part of it resembles a pear that is small in size at the top and large at the bottom, and its tissue is very similar to the spongy tissue, closed from the outside by a thin membrane called (membrane) Crystalline) which facilitates the process of the lungs sliding onto the inner surface of the rib cage. The right lung is divided into three lobes, while the left lung is divided into two lobes, and the heart represents the place of the third chapter, and each lobe is divided into two hundred lobes, both of which contain alveoli, as each lung contains On several millions of alveoli, each vesicle has a diameter of one milliliter, and one of the scientists mentioned that the alveoli is estimated to be equivalent to forty to fifty times the surface area of human skin, and the walls of these alveoli are spontaneously wet and very thin, yet they are strong and strong very

- The rib cage: it is a box located at the top of the human body between the neck and the trunk, and it consists of twenty-four ribs, of which twelve are on the right of the chest and twelve on the left of the chest and are called (the real ribs), and they fuse from the back With the spine and connected from the front to the bone of the rib cage, while the other five ribs are called the floating ribs) because they are not fixed in the rib cage from the front, especially the three short ribs, they are not fixed from the front and fixed from the back only, and it controls the movement of the ribs The chest is a thin, superficial muscle found between the ribs that stretch during inhalation. For example, the lungs do not move by themselves, and therefore the ribs do not extend by themselves, but the diaphragm is the leader responsible for the movement of these two organs (lungs / ribs).

Breathing:

A vital process that takes place through the respiratory system automatically. When we breathe, air is drawn from the nostrils and enters into the mucous membranes charged with a large amount of warm blood, passing through the nasopharynx, then the mouth, reaching the larynx, 
then the trachea, followed by the two bronchi that branch into smaller and smaller bronchioles ending in the alveoli. And we should know that the blood stays in the blood vessels only from one to two seconds, and during this little time the gases are exchanged, so the body expels carbon dioxide and receives oxygen, with a number ranging from 12-20 times per minute in normal conditions for people. Adults without any effort, in which the oxygen necessary for the body is obtained, carbon dioxide is disposed of, that a person breathes about 22 thousand times a day, and in the process of inhalation, the body withdraws about 500 cubic centimeters of air and expels Approximately the same amount, that the air enters the lungs exhaled due to the movement of the diaphragm, which works permanently like the movement of the heart. Exhalation process Th. A center in the brain called the Respiratory Control Center sends signals to the muscles responsible for the process of breathing continuously, so that it occurs automatically without feeling it, but a person can control his breathing rate to some extent, in addition to some factors that may It affects the breathing process, such as feelings and physical activity.

Types of breathing: (upper respiration - middle breathing - deep or lower breathing)

- Upper respiration: depends on the movement of the lung with the collar bone, by raising the shoulders and the collar bone in addition to the rib bones upwards, so the air rushes to the upper part of the lung, which is the smallest part in relation to the size of the lung, and thus the body benefits with a small portion of air, so this is considered Type is the worst type of breathing ever known because most people rely on this method.

- Middle breathing: depends on the movement of the muscles that connect the ribs of the rib cage, which is stretched specifically. This method is better than upper respiration and much less than deep or lower breathing. Deep or lower breathing: It is considered the best way for proper breathing, when the inhalation is deep, the air fills the lower part of the lungs, which is the largest part, so the lungs expand and expand until they touch the surface of the diaphragm, which in turn decreases until it touches the stomach wall until it touches the diaphragm, which in turn decreases. Until it touches the stomach wall and presses it, which leads to a protrusion in the abdomen, as there are ribs of the cage alveoli to the maximum extent, and it is possible to control the diaphragm muscle in order to control the process of exhaling air exit in the required amount through various breathing exercises, for example Example (for a person to lie on a flat and comfortable place or sit on a chair, close his eyes and take a breath from the nose, so that the shoulders, jaws, neck and back are in a comfortable position).

The importance of correct breathing: 
The importance of correct breathing lies in obtaining the adequate quantities of oxygen needed for the various organs of the body, in addition to the fact that the level of carbon dioxide has an important role in maintaining the required $\mathrm{pH}$ for the body, to perform its functions properly without feeling tired and exhausted, and correct breathing reduces tension, and improves It enhances the functioning of the individual's immune system, which is important to protect the body from infection and disease, and correct breathing helps prevent panic attacks and fear (Panic attacks), in addition to getting rid of insomnia and depression. Breathing through the nose ensures the required balance between oxygen and carbon dioxide inside the body and maintains the level of oxygen entering the cells.

1- Resorting to non-breathing through retention of the process of inhalation and exhalation, which makes beginners in a state of anxiety, tension, and lack of focus during the musical performance, and vice versa.

2- The shoulder is slightly raised with severe pressure on all joints, starting from (arm - elbow - wrist - fingers), in addition to the muscles, which leads to difficulty controlling the fingers of the piano keyboard.

3- Feeling severe pain in the area of the neck muscles as a result of severe pressure on the cervical vertebrae and failure to breathe correctly and regularly helps to relax the muscles. Proper control without effort.

4- Curvature of the upper part of the back (the spine) and a rise upwards, forming a semicircle with the head and neck tilted slightly forward (the correct position, the straightness of the arms leaving freedom of movement on the piano keyboard), which causes severe pressure on the neck and joints associated with musical performance that began with a joint The shoulder and ending with the finger joints.

5- Failure to follow the correct session in front of the piano keyboard in the middle on the chair designated for that, with the feet not touching the ground and therefore not forming a right angle with stability for the comfort of the body and the student becomes in a state of psychological and physiological pressure.

6- Inability to place the hand and number the fingers correctly in the vocal layers recorded in the musical notes.

7 - The appearance of signs of distraction and lack of focus in eye movement, which leads to errors in reading the musical rhythmic and melody.

8- Excessive movements of the wrist joint, either up or down, with the palm of the hand lead to difficulty controlling and balancing between the fingers and toes of the piano keyboard. 
9- Training intermittently affects the concentration of beginners, especially solfege, using simple scales, because it is the basis of musical reading (melodic and rhythmic) on the piano, with reliance on taking out the pieces by listening only without learning the musical language. 10- A little beginners lack a balance between the different senses used in the performance and the process of breathing regularly because it forms a rhythm somewhat similar to the rhythm of performing a piece of music.

11- Beginners are afraid of making a loud sound during the breathing process, which causes them anxiety and tension.

12- Beginners use their fingers randomly without adhering to the correct numbering, which leads to inconsistency in playing the piano keyboard.

Verify the second question:

What are the suggested exercises that involve performing well on the psyche? The beginners' sense of correct natural breathing during the performance, which resulted in rest, focus and control of the fingers of the piano keyboard because the body got enough oxygen that helped to express the sound music, where the auditory, visual and motor memory (muscle memory) interacts to serve skills techniques Music performance in addition to the senses (soul performance in musical phrases and sentences) on a piano.

The educator and the novice must also interact with each other through listening - interpretation and simplicity skills - actual performance - careful observation - immediate correction of errors with the facilitated explanation - adjusting the performance by playing a model on the piano keyboard - correct execution of the previously explained steps - encouragement and praise Adjustment and guiding beginners to achievement, advancement, and self-confidence for better artistic and expressive musical performance. The first and second exercises are designed away from the central area - the piano keyboard helps students to take the correct position to sit in front of the keyboard to give the beginners a good opportunity to control and focus on taking their breath and performing correctly and in balance. This was also confirmed by Qigong through several steps as a guideline for linking mental awareness with breathing while performing on the piano:

The first step: for the novice to be in the correct position to sit in front of the piano keyboard, with feet steady on the floor, resting shoulders and arms, and placing hands on the knees, close your eyes - take a few deep breaths with slow breathing down to the stomach / abdomen area without pressure first ( Inhalation (inhalation with a capacity of approximately $70 \%$ before the chest and lung is filled with air ") with the chest and lung filled, the breaths are expelled by emptying the lungs first, then the abdomen (exhalation). 
The second step: for the beginner to begin performing the role of the grand ladder of two octaves from the bottom of the middle du tone with the left hand, provided that the arm is free to move with attention to breathing regularly and smoothly.

The third step: When the novice reaches the highest point in obtaining air by about $70 \%$, make your left hands perform a great triple composition on the du tone with equal touch, then combine the right hand with the role of the big ladder, the number of two octaves of the middle Du tone, while continuing to escape The breath slowly.

The fourth step: The third step is repeated, then the novice performs the triple synthesis with the right hand and the left hand alternately while breathing deeply, freely and slowly in inhalation and exhalation - determining, monitoring and following your breathing speed with the speed of the exercise without controlling it. Andras Schift also emphasized that natural breathing leads to the absence of tension or pain in the back and wrists, and that the beginner must be aware of the importance of breathing and notice it during the performance so that the beginner becomes more fluent to revive the music presented through

Piano tracks / exercises.

Recommendations and proposals:

1- Pre-preparation by preparing and educating beginners on the importance of using correct breathing exercises before starting an actual playing performance on the piano.

2- Training on attention, focus and follow-up in the breathing process and how it relates to controlling performance

\section{References}

1- Amal Sadiq Fouad Abu Hatab: Educational Psychology, The Anglo-Egyptian Library, Cairo, Sixth Edition 2000 AD.

2- Jaber Abdel-Hamid, Ahmed Khairy Kazem: "Research Methods in Education and Psychology", Dar Al-Nahda Al-Arabiya, Cairo 2002.

3- Inayat Ahmed Wasfi: Memoirs of Singing Teaching Methods, Faculty of Music Education, Helwan University, 1993 AD.

4- Rosalind Ryan: "How You Can Breathe Your Way to Good Health", www.dailymail.co.uk, Retrieved, Edited 25-2-2018.

5- Christine Evans: "The Importance of Breathing Through Your Nose", Edited(1-7-2016), Retrieved 3-2-2018., www.healthyplace.com.

6- William Blahd: "How the Lungs and Respiratory System Work” “www.webmd.com , Retrieved 3-2-2018. Edited (14-7-2017).

7- Payne, R.A. Relaxation Techniques: A Practical Handbook for the Health Care Professional. 
London: Churchill Livingstone, (2000).

8- Yutaka Sakaguchi \& Eeiko Aiba : " Relationship between Musical Characteristics and Temporal Breathing Pattern in Piano Performance" „research paper, university of Electrocommunications ,Chofu , Japan, copyright Sakaguchi \& Aiba ,2016. https://www.ncbi.nlm.nih.gov/pmc/?term=Breathing+Pattern+in+learning++Piano+Perfoima nce

9- Ming Shiow Huang : " Coping with Performance Anxiety: College Piano Students' Perceptions of Performance Anxiety and Potential Effectiveness of Deep Breathing, Deep Muscle Relaxation, and Visualization", Florida State University -college of music ,PHD ,2011. 10- Agnes S.Chan ,Mei-Chun Cheung, Sophia L. Sze, Winnie Wing-Man Leung, Dejian Shi : Shaolin Dan Tian Breathing Fosters Relaxed and AttentiveMind: A Randomized Controlled Neuro-Electrophysiological Study, The Chinese University of Hong Kong, New Territories, Hong Kong, 2010-2011 https://www.ncbi.nlm.nih.gov/pmc/articles/PMC2957109/

11- https://pianodao.com/2016/11/02/breathing-piano/

https://fundamentals-of-pianopractice.readthedocs.io/en/latest/ chapter1/ch1_procedures/11.21.html

13- https://www.ncbi.nlm.nih.gov/pmc/articles/PMC4963403/

14- https://en.wikipedia.org/wiki/Breath_mark,VirginiaTech Multimedia Music Dictionary article on pause markings, (Accessed 20 April 2006), last edited on 8 March 2018, at 18:28 (UTC) 14- https://musicterms.artopium.com/b/Breathmark.htm , 2018.

15-https://www.lds.org/bc/content/shared/english/pdf/callings/music/keyboard course/33620_eng.pdf?lang =eng Basic (Music Course - Keyboard Course: Published by The Church of Jesus Christ of Latter-day Saints Salt Lake City, Utah - Intellectual Reserve 1993, Inc. All rights reserved \& Printed in USA, Update 2004.)

16- https://pianodao.com/2018/04/02/more-breathing-at-the-piano/ $17-$

https://www.education.vic.gov.au/Documents/school/principals/management/voicetechniques .pdf

18- https://www.onlinepianocoach.com/musical-phrasing

Received: February 5, 2018

Accepted: April 2, 2018 